\title{
Growth, Structural and Optical Properties of GaAs/AlGaAs Core/Shell Nanowires with and without Quantum Well Shells
}

\author{
Hannah J. Joyce*, Y. Kim, Q. Gao, H. H. Tan, C. Jagadish \\ Department of Electronic Materials Engineering, Research School of Physical Sciences and Engineering, \\ The Australian National University, Canberra, ACT 0200, Australia \\ *Email: hjj109@rsphysse.anu.edu.au
}

\begin{abstract}
We have investigated the growth, structural properties and photoluminescence of novel GaAs/AIGaAs radial heterostructure nanowires, fabricated by metalorganic chemical vapour deposition. The effect of growth temperature on nanowire morphology is discussed. Strong photoluminescence is observed from GaAs nanowires with AlGaAs shells. Core/multishell nanowires, of GaAs cores clad in several alternating layers of thick AlGaAs barrier shells and thin GaAs quantum well shells, exhibit a blue-shifted photoluminescence peak believed to arise from quantum confinement effects. A novel two-temperature growth procedure for obtaining GaAs cores is introduced, and other nanowire heterostructures are addressed.
\end{abstract}

Keywords-nanowire; GaAs; AlGaAs; heterostructure; photoluminescence.

\section{INTRODUCTION}

Semiconductor nanowires have a wide range of potential applications, and have consequently attracted much research interest in recent years $[1,2]$. In particular, III-V nanowires are promising nano-building blocks for future optoelectronic devices. These nanowires can be epitaxially grown by metalorganic chemical vapour deposition (MOCVD) [3]. In this growth process, nanometre-scale gold particles promote anisotropic wire-like growth by either a vapour-liquid-solid (VLS) or vapour-solid-solid (VSS) mechanism [3, 4]. At growth temperature, the gold nanoparticles on the semiconductor substrate surface form a liquid (for VLS) or solid (for VSS) alloy with the group III species. These nanoparticles are ideal sinks for group III species supplied from the surrounding vapour, and are readily supersaturated. As the group III species precipitate out at the nanoparticlesemiconductor interface, highly anisotropic nanowire growth takes place, with a nanoparticle atop each nanowire.

Radial heterostructure nanowires will play a major role in future nanowire-based optoelectronic devices. Nanowire heterostructures with quantum well shells have been demonstrated [5], and functional devices such as high efficiency LEDs have been fabricated from core/shell nanowires [6]. Study of GaAs/AlGaAs radial heterostructures should prove important in the development of nanowire technology. MOCVD has been used previously to fabricate GaAs and AlGaAs nanowires on GaAs (111)B substrates [3, 7,
8]. Although GaAs/AlGaAs core/shell nanowires have been grown by selective area MOCVD [9], Au nanoparticle-assisted growth of these structures by MOCVD is yet to be reported. Additionally, GaAs/AlGaAs core/multishell nanowires are yet to be reported. In this paper, we investigate the Au-assisted growth and optical properties of GaAs/AlGaAs core/shell and core/multishell nanowires, including nanowires with GaAs quantum well shells.

Nanowire tapering and kinking are two growth issues which must be overcome to obtain uniform GaAs cores with good crystallographic and optoelectronic properties. Growth temperature affords control over nanowire morphology. A twotemperature growth procedure is proposed to minimise tapering and kinking of GaAs nanowire cores. In addition, MOCVD growth of axial heterostructure nanowires is discussed.

\section{EXPERIMENTS}

Undoped GaAs (111)B substrates were functionalised by immersion in $0.1 \%$ poly-L-lysine (PLL) solution for $1 \mathrm{~min}$. A $100 \mu \mathrm{L}$ droplet of gold colloid solution $(10,20,30$ or $50 \mathrm{~nm}$ diameter $\mathrm{Au}$ particles at a concentration of approximately $10^{11}$ particles $/ \mathrm{ml}$ ) was applied to the substrate, then rinsed off after 10 seconds in contact. The negatively charged gold nanoparticles are attracted to, and immobilised on, the positively charged PLL layer on the substrate surface. Nanowires, catalysed by these particles, were grown by horizontal flow MOCVD at a pressure of $100 \mathrm{mbar}$. Trimethylgallium (TMGa, $1.2 \times 10^{-5} \mathrm{~mol} / \mathrm{min}$ ) and $\mathrm{AsH}_{3}$ $\left(5.4 \times 10^{-4} \mathrm{~mol} / \mathrm{min}\right)$ were precursors for $\mathrm{GaAs}$ core and shell growth. AlGaAs shells were grown by introducing TMAl $\left(4.1 \times 10^{-6} \mathrm{~mol} / \mathrm{min}\right)$, with the same $\mathrm{TMGa}$ and $\mathrm{AsH}_{3}$ flows.

Prior to nanowire growth initiation, the substrate was annealed under arsine pressure at $600^{\circ} \mathrm{C}$ to desorb surface contaminants. Growth temperatures between 390 and $510^{\circ} \mathrm{C}$ were tested to determine the optimum growth conditions for GaAs nanowire cores. For core/shell heterostructures, GaAs core growth was carried out at $450^{\circ} \mathrm{C}$. Shells of GaAs and $\mathrm{AlGaAs}$ were grown at $650^{\circ} \mathrm{C}$. A two-temperature procedure was trialed for GaAs core growth, involving a nucleation step under TMGa flow at $450^{\circ} \mathrm{C}$, cooling under arsine pressure to $390^{\circ} \mathrm{C}$, then growth at $390^{\circ} \mathrm{C}$ under TMGa flow. 
Nanowire superlattices of GaAs/InGaAs and GaAs/AlGaAs were grown at $450^{\circ} \mathrm{C}$. Axial InGaAs segments were grown with TMIn flows up to $3.5 \times 10^{-5} \mathrm{~mol} / \mathrm{min}$, and TMGa and $\mathrm{AsH}_{3}$ flows described above, as used previously for InGaAs nanowire growth [10]. Axial AlGaAs segments were grown with TMAl, TMGa and $\mathrm{AsH}_{3}$ flows as used for $\mathrm{AlGaAs}$ shells.

Nanowire structure was examined using field emission scanning electron microscopy (FE-SEM) with an accelerating voltage of $3 \mathrm{kV}$. Nanowire photoluminescence (PL) spectra and micro-photoluminescence (micro-PL) images were measured. For both these measurements, nanowires were excited with the $532 \mathrm{~nm}$ line of a frequency-doubled diodepumped solid-state (DPSS) laser. PL spectral measurements were performed at low temperature $(10 \mathrm{~K})$, with the PL signal dispersed through a $0.5 \mathrm{~m}$ monochromator then detected by a thermoelectrically cooled InGaAs photodetector. Micro-PL images were obtained at room temperature using an epifluorescence microscope (Nikon Eclipse L150). The excitation laser spot was focused onto the sample by a 0.8 numerical aperture, $\times 50$ objective lens (Nikon CFI LU Plan Epi). PL was collected through this objective and micro-PL images were captured using a Peltier-cooled CCD camera (Nikon DS-5Mc).

\section{RESUlTS AND DISCUSSION}

\section{A. Growth of Nanowire Cores and Shells}

Nanowires were generally aligned in the [111] direction on GaAs (111)B substrates. The crystallographic quality of these nanowires is inferred from their hexagonal and triangular cross-sections.

Nanowire morphology was found to be strongly dependent on growth temperature. Figure $1 \mathrm{a}, \mathrm{b}$ and $\mathrm{c}$ shows FE-SEM images of nanowires grown at $510^{\circ} \mathrm{C}, 450^{\circ} \mathrm{C}$ and $390^{\circ} \mathrm{C}$ respectively, and Figure 1d shows the dependence of axial and radial growth rates on growth temperature. The axial growth rate peaks at $480^{\circ} \mathrm{C}$ but the radial growth rate increases with increasing growth temperature. This radial growth induces significant tapering with increasing severity at higher growth temperatures, as in Figure 1a. Nanowires grown at lower temperatures feature relatively uniform diameters but suffer from kinking, as in Figure 1c. An optimised growth temperature of $450^{\circ} \mathrm{C}$, chosen to minimise tapering and kinking simultaneously, was used for the growth of all nanowire cores in core/shell nanowires.
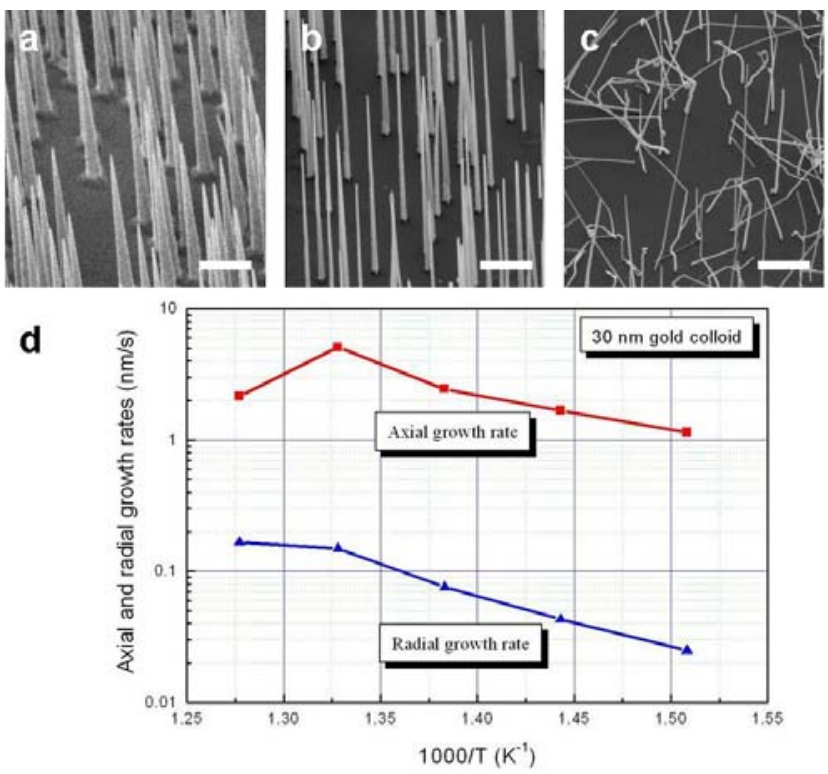

Figure 1. FE-SEM images of nanowires catalysed by $30 \mathrm{~nm}$ diameter $\mathrm{Au}$ particles and grown for 30 minutes at a) $510^{\circ} \mathrm{C}$, b) $450^{\circ} \mathrm{C}$ and c) $390^{\circ} \mathrm{C}$. Samples are tilted at $45^{\circ}$. Scale bars are $1 \mu \mathrm{m}$. d) Temperature dependence of axial and radial growth rates for GaAs nanowires.

As the radial growth rate increases with increasing temperature, the growth mode can be effectively switched from axial nanowire growth to lateral shell overgrowth by increasing the temperature. Therefore, GaAs and AlGaAs shells were grown at $650^{\circ} \mathrm{C}$.

\section{B. Core/Shell Heterostructures and Photoluminescence}

Core/shell $\mathrm{GaAs} / \mathrm{AlGaAs}$ heterostructures are possible because the lattice constants of GaAs and AlGaAs are effectively identical. Figure 2a illustrates GaAs nanowires grown for 30 minutes, then clad in AlGaAs. A schematic is included in Figure $2 \mathrm{~b}$. These GaAs nanowires are presumably passivated by their AlGaAs shells, and exhibited strong GaAsrelated PL.

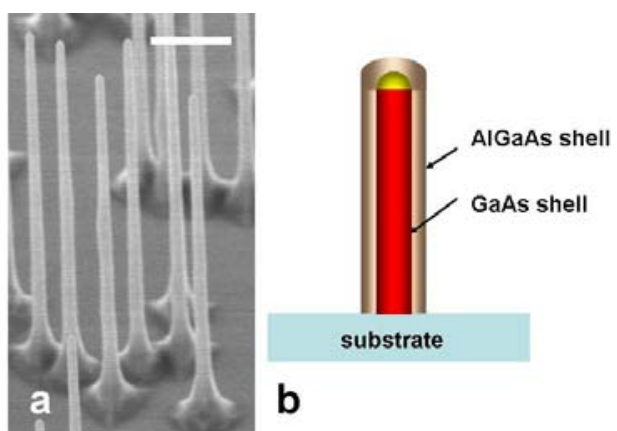

Figure 2. FE-SEM image of GaAs nanowires catalysed by $30 \mathrm{~nm}$ diameter Au particles, grown for 30 minutes at $450{ }^{\circ} \mathrm{C}$, then clad in a shell of AlGaAs at $650^{\circ} \mathrm{C}$. Scale bar is $1 \mu \mathrm{m}$. (b) Schematic of a core/shell nanowire.

Figure 3a illustrates GaAs nanowires grown for 10 minutes. Figure $3 \mathrm{~b}$ illustrates similarly grown $\mathrm{GaAs}$ nanowires encased in shells by growth of AlGaAs (10 minutes), GaAs (15 s), AlGaAs (2 minutes), GaAs (10 s), AlGaAs (2 minutes), GaAs (6s) and AlGaAs (3 minutes). Such thin GaAs shells surrounded by AlGaAs barriers, as grown here, are expected to 
behave as quantum well shells. A schematic of a nanowire with a single quantum well shell is shown in Figure 3c. Indeed, low temperature PL measurements of the nanowires exhibit a blueshifted peak at $1.61 \mathrm{eV}$, additional to the GaAs core-related peaks at around $1.5 \mathrm{eV}$ (Figure 3d). This suggests quantum confinement in at least one GaAs shell. Further investigations are underway to assess the relationship between GaAs shell growth time and PL blue shift.

Room temperature micro-PL measurements confirm the strong PL arising from these nanowires. PL is predominantly emitted from nanowire ends, owing to the wave-guiding nature of the wires. Figure 3e shows PL from four nanowires, viewed from the tips of these nanowires as-grown on the original $\mathrm{GaAs}$ substrate. The substrate exhibits no measurable signal within the micro-PL system wavelength sensitivity range. One possible explanation is that GaAs and AlGaAs shell growth over nanowires occurs at a greater rate than substrate overgrowth. This would be an interesting growth anomaly and is to be confirmed by further investigation. Another possibility is that wave-guiding along each nanowire results in enhanced emission from each nanowire tip, relative to the substrate. A final factor is that nanowires have a larger surface area than the substrate, leading to a stronger PL signal.
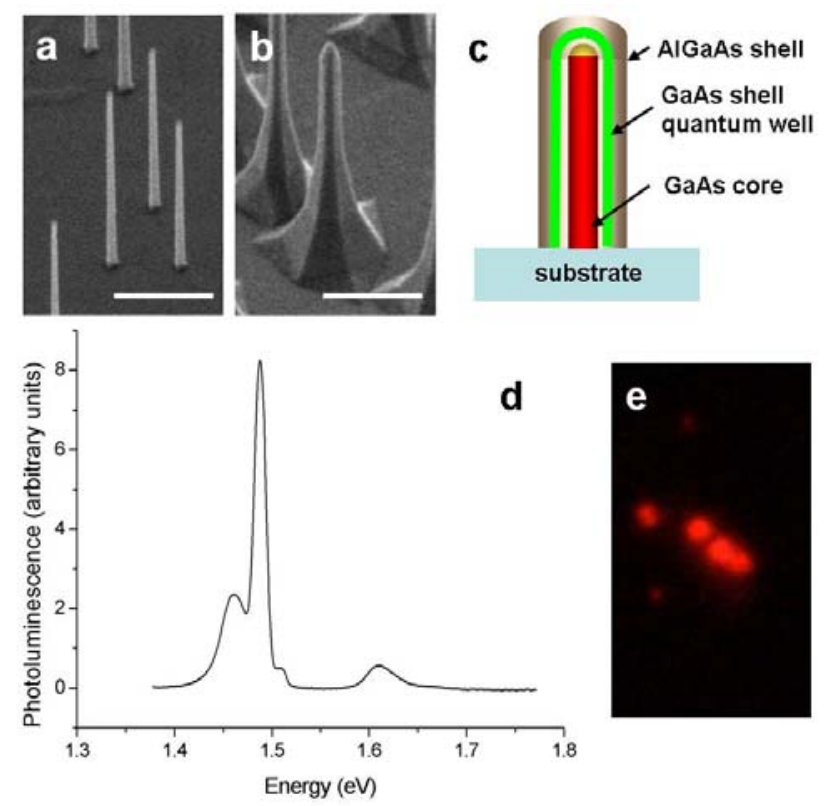

Figure 3. a) FE-SEM image of GaAs nanowires catalysed by $50 \mathrm{~nm}$ diameter Au particles and grown for 10 minutes. b) FE-SEM image of GaAs nanowires clad with alternating AlGaAs barrier shells and GaAs shell quantum wells. Samples are tilted at $45^{\circ}$. Scale bars are $1 \mu \mathrm{m}$. c) Schematic of a GaAs nanowire with a radial GaAs quantum well. d) PL spectrum taken at $10 \mathrm{~K}$ from nanowires in b). e) Micro-PL image of nanowires in b), as-grown on the GaAs (111)B substrate.

Core/multishell nanowires were grown with GaAs core (20 minutes), AlGaAs shell (15 minutes), GaAs shell ( 3 minutes) and AlGaAs cladding (10 minutes). Figure $4 \mathrm{a}$ shows the optical image of these nanowires transferred to a silicon substrate and Figure $4 \mathrm{~b}$ shows the micro-PL image of the central nanowire, excited by a focused laser spot. PL is emitted along the complete length of the nanowire. Polarisation dependence of nanowire PL is also observed. Maximum PL is observed when the excitation light is polarised parallel to the nanowire, while minimum PL occurs when the excitation light is polarised perpendicular to the nanowire.

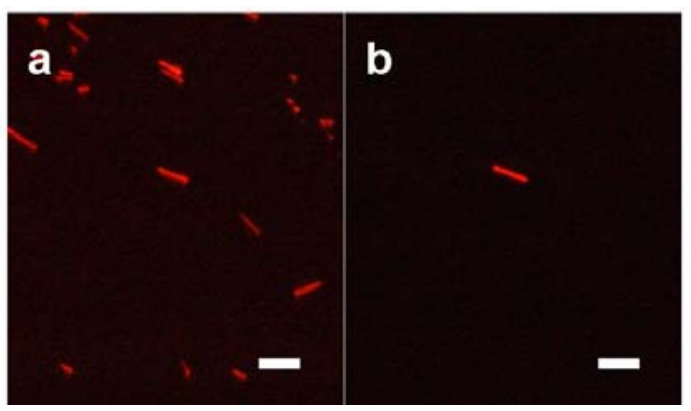

Figure 4. a) Optical image of core/shell nanowires transferred to a silicon substrate. b) Micro-PL image of a single nanowire excited by a focused laser spot. This nanowire corresponds to the central nanowire in a). Scale bars are $5 \mu \mathrm{m}$.

\section{Optimisation of Nanowire Core Growth}

A two-temperature growth procedure, involving a nucleation step at high temperature followed by growth at a lower temperature, is currently being trialled to further improve GaAs nanowire core morphology. This is similar to a procedure developed for Ge nanowires [11]. The nucleation step was performed under TMGa flow at $450^{\circ} \mathrm{C}$ for 1 minute, in order to form the supersaturated $\mathrm{Au} / \mathrm{Ga}$ nanoparticle alloy and promote nucleation at the substrate/nanoparticle interface. TMGa flow was removed during cooling to $390^{\circ} \mathrm{C}$, and then reintroduced to resume nanowire growth at $390^{\circ} \mathrm{C}$. Nanowires grown by this procedure are illustrated in Figure 5. These nanowires have a more uniform diameter than those grown at a single temperature of $450^{\circ} \mathrm{C}$ (Figure 1b). Nanowire kinking occurs less frequently compared with nanowires grown at a single temperature of $390^{\circ} \mathrm{C}$ (Figure 1c). Thus kinking and tapering are simultaneously minimised by the two-temperature procedure.

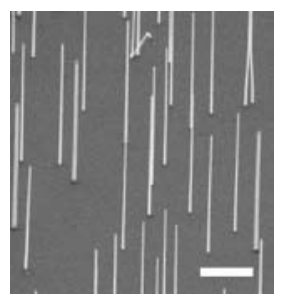

Figure 5. Nanowires grown using a two-temperature procedure with a 1 minute nucleation step at $450^{\circ} \mathrm{C}$ followed by 30 minutes growth at $390^{\circ} \mathrm{C}$ Scale bar is $1 \mu \mathrm{m}$.

\section{Other Heterostructures}

Nanowires permit radial expansion and contraction of the crystal lattice, allowing axial heterostructures of highly lattice mismatched materials. Thus nanowires offer greater flexibility in materials systems than conventional planar growth. For example, thin $(<10 \mathrm{~nm})$ InGaAs segments may be grown in GaAs nanowires, and are expected to behave as axial quantum wells. MOCVD growth of novel GaAs/InGaAs and $\mathrm{AlGaAs} / \mathrm{GaAs}$ nanowire superlattices is reported here. 
Nanowires with GaAs/InGaAs superlattices have been successfully grown by MOCVD, as illustrated in Figure 6 . Ten InGaAs segments were grown with a TMIn vapour mole fraction $\left(\mathrm{x}_{\mathrm{v}}, \mathrm{TMIn}\right)$ of 0.23 for $1 \mathrm{~s}$, sandwiched between barriers of GaAs grown for 1 minute each. Growth interrupts of 2 minutes were used between switching Group III flows, to deplete the Au nanoparticle of the previous Group III species. These nanowires remain vertically oriented despite the introduction of InGaAs segments. Nanowire kinking occurred more frequently when smaller Au nanoparticles were used, and when higher TMIn flow was used.

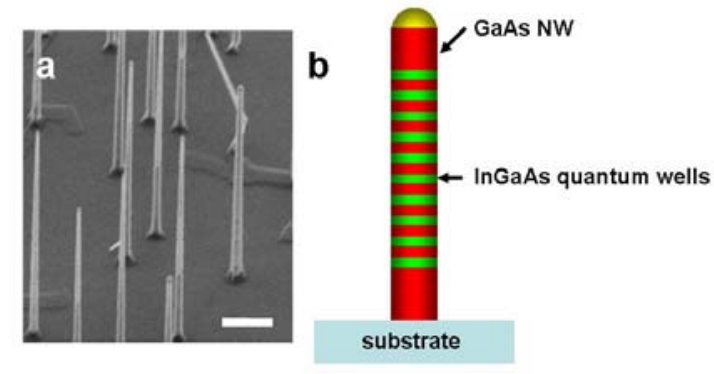

Figure 6. a) FE-SEM image of GaAs/InGaAs superlattice nanowires, catalysed by $50 \mathrm{~nm}$ diameter Au particles. Scale bar is $1 \mu \mathrm{m}$. b) Schematic of

a GaAs/InGaAs superlattice nanowire with ten InGaAs quantum wells.

Superlattices of AlGaAs/GaAs are typically kinked, despite the lattice matching of the $\mathrm{AlGaAs}$ and GaAs materials systems. It is thought that oxygen gettering and lowtemperature growth of $\mathrm{AlGaAs}$ contribute to the imperfect morphology of these nanowires.

\section{CONCLUSION}

MOCVD was used to fabricate GaAs nanowires and $\mathrm{GaAs} / \mathrm{AlGaAs}$ radial heterostructure nanowires. Nanowire growth was sensitive to growth temperature, with higher temperatures leading to tapering and lower temperatures resulting in irregular kinked morphologies. Strong PL is observed from GaAs nanowires clad in, and presumably passivated by, AlGaAs shells. Core-multishell nanowires, of GaAs cores clad in several alternating layers of thick AlGaAs barrier shells and thin GaAs quantum well shells, exhibit a blue-shifted photoluminescence peak. This blue-shift is thought to arise from quantum confinement effects in one of the GaAs shells.
We have devised a novel two-temperature growth procedure for obtaining straight, well-oriented epitaxial GaAs cores with minimal tapering. In addition to radial heterostructures, axial heterostructure nanowires such as $\mathrm{GaAs} / \mathrm{InGaAs}$ superlattices were successfully fabricated by MOCVD.

\section{ACKNOWLEDGEMENT}

The Australian Research Council is gratefully acknowledged for its financial support.

\section{REFERENCES}

[1] Y. N. Xia, P. D. Yang, Y. G. Sun, Y. Y. Wu, B. Mayers, B. Gates, Y. D. Yin, F. Kim, and Y. Q. Yan, "One-dimensional nanostructures: Synthesis, characterization, and applications," Adv. Mater., vol. 15, pp. 353-389, 2003.

[2] C. M. Lieber, "Nanoscale science and technology: Building a big future from small things," MRS Bull., vol. 28, pp. 486-491, 2003.

[3] K. Hiruma, M. Yazawa, K. Haraguchi, K. Ogawa, T. Katsuyama, M. Koguchi, and H. Kakibayashi, "GaAs freestanding quantum-size wires," J. Appl. Phys., vol. 74, pp. 3162-3171, 1993.

[4] K. A. Dick, K. Deppert, L. S. Karlsson, L. R. Wallenberg, L. Samuelson, and W. Seifert, "A new understanding of Au-assisted growth of III-V semiconductor nanowires," Adv. Funct. Mater., vol. 15, pp. 1603-1610, 2005 .

[5] P. Mohan, J. Motohisa, and T. Fukui, "Fabrication of InP/InAs/InP coremultishell heterostructure nanowires by selective area metalorganic vapor phase epitaxy," Appl. Phys. Lett., vol. 88, 2006.

[6] F. Qian, S. Gradecak, Y. Li, C.-Y. Wen, and C. M. Lieber, "Core/multishell nanowire heterostructures as multicolor, highefficiency light-emitting diodes," Nano Lett., vol. 5, pp. 2287-2291, 2005.

[7] Z. H. Wu, M. Sun, X. Y. Mei, and H. E. Ruda, "Growth and photoluminescence characteristics of AlGaAs nanowires," Appl. Phys. Lett., vol. 85, pp. 657-659, 2004.

[8] K. Hiruma, M. Yazawa, T. Katsuyama, K. Ogawa, K. Haraguchi, M. Koguchi, and H. Kakibayashi, "Growth and optical properties of nanometer-scale GaAs and InAs whiskers," J. Appl. Phys., vol. 77, pp. 447-462, 1995.

[9] J. Noborisaka, J. Motohisa, S. Hara, and T. Fukui, "Fabrication and characterization of freestanding $\mathrm{GaAs} / \mathrm{AlGaAs}$ core-shell nanowires and AlGaAs nanotubes by using selective-area metalorganic vapor phase epitaxy," Appl. Phys. Lett., vol. 87, 2005.

[10] Y. Kim, H. J. Joyce, Q. Gao, H. H. Tan, C. Jagadish, M. Paladugu, J. Zou, and A. A. Suvorova, "Influence of nanowire density on the shape and optical properties of ternary InGaAs nanowires," Nano Lett., vol. 6, pp. 599-604, 2006.

[11] H. Adhikari, P. C. McIntyre, S. Y. Sun, P. Pianetta, and C. E. D. Chidsey, "Photoemission studies of passivation of Germanium nanowires," Appl. Phys. Lett., vol. 87, 2005. 